The Journal of Nonlinear $\mathbf{S}_{\text {cience and Applications }}$ http://www.tjnsa.com

\title{
EXISTENCE AND MULTIPLICITY OF POSITIVE SOLUTIONS FOR A $p$-LAPLACIAN BOUNDARY VALUE PROBLEM ON TIME SCALES
}

\author{
YUANYUAN PANG ${ }^{1, *}$ AND ZHANBING BAI ${ }^{2}$
}

\begin{abstract}
In this paper, we study the solvability of one-dimensional fourthorder $p$-Laplacian boundary value problems on time scales. By using Krasnosel'skii's fixed point theorem of cone expansion-compression type, some existence and multiplicity results of positive solution have been required according to different growth condition of nonlinear form $f$ at zero and at infinity.
\end{abstract}

\section{INTRODUCTION}

The study of dynamic equations on time scales goes back to its founder Stefan Hilger [14], and is a new area of still fairly theoretical exploration in mathematics. Recently, there has been much attention paid to the existence of positive solutions for second-order nonlinear boundary value problems on time scales, see $[1,5,11]$. On the one hand, the higher-order nonlinear boundary value problems have been studied extensively, see $[4,9,12,16]$. On the other hand, the boundary value problems with $p$-Laplacian operator have also been discussed extensively in the literature, for example, see $[6,17,18,20]$. However, to the best of our knowledge, there are not many results concerning the fourth-order $p$-Laplacian dynamic equations on time scales.

In [10], Bai and Du considered the following second-order four-point boundary value problems

$$
\left\{\begin{array}{l}
-x^{\prime \prime}+\lambda h(t) f(t, x(t))=0, \quad 0<t<1, \\
x(0)=a x(\xi), \quad x(1)=b x(\eta)
\end{array}\right.
$$

Date: Received: 3 March 2009; Revised: 13 June 2009.

* Corresponding author.

2000 Mathematics Subject Classification. Primary 34B15; Secondary 34B18.

Key words and phrases. Time scales; $p$-Laplacian operator; Positive solution; Cone. 
where $0<\xi<\eta<1,0 \leq a, b<1$, and $h:[0,1] \times[0, \infty) \rightarrow[0, \infty)$ are nonnegative continuous functions. The authors established the existence, nonexistence, and multiplicity of positive solutions by using the fixed-point index theory, LeraySchauder degree and the upper and lower solution method.

In [19], Wang studied the following boundary value problem

$$
\left\{\begin{array}{l}
\left(g\left(u^{\prime}\right)\right)^{\prime}+a(t) f(u)=0, \quad 0<t<1, \\
u(0)=0, \quad u(1)=0,
\end{array}\right.
$$

where, $g(v):=|v|^{p-2} v, p>1$. It shows the existence of one positive solution of this problem by used Cone compression-extension theorem, if

$$
f_{0}=0, f_{\infty}=+\infty, \text { or } f_{0}=+\infty, f_{\infty}=0,
$$

where

$$
f_{0}=\lim _{u \rightarrow 0} \frac{f(u)}{u^{p-1}}, f_{\infty}=\lim _{u \rightarrow \infty} \frac{f(u)}{u^{p-1}} .
$$

Ma [16] proved the existence of positive solutions of the following beam equation under above conditions

$$
\left\{\begin{array}{l}
u^{(4)}+a(t) f(u)=0, \quad 0<t<1, \\
u(0)=u(1)=u^{\prime \prime}(0)=u^{\prime \prime}(1)=0 .
\end{array}\right.
$$

In [17], Sun and Li discussed the following p-laplacian m-point BVP on time scales:

$$
\left\{\begin{array}{l}
\left(\varphi_{p}\left(u^{\Delta}(t)\right)^{\nabla}+a(t) f(t, u(t))=0, \quad t \in(0, T),\right. \\
u(0)=0, \quad \varphi_{p}\left(u^{\Delta}(T)\right)=\sum_{i=1}^{m-2} a_{i} \varphi_{p}\left(u^{\Delta}\left(\xi_{i}\right)\right) .
\end{array}\right.
$$

The authors obtained some new results for the existence of at least twin or triple positive solutions by applying Avery-Henderson and Leggett-Williams fixed point theorems respectively.

In this paper, under the assumptions

$$
f_{0}=f_{\infty}=+\infty \text { or } f_{\infty}=f_{0}=0,
$$

we are concerned with the existence and multiplicity results of positive solutions for the following fourth-order $p$-Laplacian boundary value problem on time scales

$$
\left\{\begin{array}{l}
\left(\varphi_{p}\left(u^{\Delta \Delta}\right)\right)^{\Delta \Delta}+\lambda a(t) f(u)=0, \quad t \in(0, T), \\
u(0)=u(T)=u^{\Delta \Delta}(0)=u^{\Delta \Delta}(T)=0,
\end{array}\right.
$$

where $\varphi_{p}(s)$ is $p$-Laplacian operator, i.e., $\varphi_{p}(s)=|s|^{p-2} s, p>1, \lambda>0$ is a parameter.

A time scales $\mathbb{T}$ is a nonempty closed subset of $\mathbb{R}$. We make the blanket assumption that $0, T$ are points in $\mathbb{T}$, and define the interval in $\mathbb{T},[0, T]:=\{t \in \mathbb{T}: 0 \leq$ $t \leq \mathbb{T}\}$. Other types of intervals are defined similarly. 
The rest of the paper is arranged as follows. We state some basic time scale definitions and some preliminary results devoted to some existence and multiplicity results of positive solution of BVP (1.1), the main tool being the cone compression-extension theorem.

\section{Some Definitions And Lemmas}

To prove the main results in this paper, we will employ several definitions and lemmas, which are based on the BVP (1.1) and can be found in $[2,8,13]$.

Definition 2.1. A time scales $\mathbb{T}$ is a nonempty closed subset of $\mathbb{R}$. For $t<\sup \mathbb{T}$, define the forward jump operator $\sigma$ :

$$
\sigma(t)=\inf \{\tau \in \mathbb{T} \mid \tau>t \in \mathbb{T}\}
$$

If $\sigma(t)>t$, $\mathrm{t}$ is said to be right scattered, and if $\sigma(t)=t, \mathrm{t}$ is said to be right dense. If $\mathbb{T}$ has a right scattered minimum $\mathrm{m}$, define $\mathbb{T}_{k}=\mathbb{T}-m$; otherwise set $\mathbb{T}_{k}=\mathbb{T}$. The forward graininess is $\mu(t)=\sigma(t)-t$.

Definition 2.2. For $f: \mathbb{T} \rightarrow \mathbb{R}$ and $t \in \mathbb{T}^{k}$, the delta derivative of $f$ at $t$ , denoted by $f^{\Delta}(t)$, is the number (provided it exists) with the property that given any $\epsilon>0$, there is a neighborhood $U \subset \mathbb{T}$ of $t$ such that

$$
\left|f(\sigma(t))-f(s)-f^{\Delta}(t)[\sigma(t)-s]\right| \leq \epsilon|\sigma(t)-s|, \quad \forall s \in U .
$$

Definition 2.3. If $F^{\Delta}(t)=f(t)$, then we define the delta integral by

$$
\int_{a}^{b} f(s) \Delta s=F(b)-F(a) .
$$

Lemma 2.4. ([15]) Let $B$ be a Banach space and $P \subset B$ be a cone in $B$. Assume $\Omega_{1}, \Omega_{2}$ are open subsets of $B$ with $0 \in \Omega_{1}, \bar{\Omega}_{1} \subset \Omega_{2}$, and let $T: P \cap\left(\bar{\Omega}_{2} \backslash \Omega_{1}\right) \rightarrow P$ be a completely continuous operator such that either

(i) $\|T u\| \leq\|u\|, u \in P \cap \partial \Omega_{1}$; and $\|T u\| \geq\|u\|, u \in P \cap \partial \Omega_{2}$, or

(ii) $\|T u\| \geq\|u\|, u \in P \cap \partial \Omega_{1}$; and $\|T u\| \leq\|u\|, u \in P \cap \partial \Omega_{2}$,

then $T$ has a fixed point in $P \cap\left(\bar{\Omega}_{2} \backslash \Omega_{1}\right)$.

Now, let the Banach space $E=C[0, T]$ be endowed with the norm $\|u\|=$ sup $|u(t)|$, and choose the cone $P \subset E$ defined by $t \in[0, T]$

$$
P=\{u \in E: u(t) \geq 0 \text { for } t \in[0, T] \text { and } u(0)=u(T)=0, u(t) \text { is concave }\} .
$$

Lemma 2.5. ([1' $]$ ) If $u \in P$, then $u(t) \geq \frac{t}{T}\|u\|$, for $t \in[0, T]$.

\section{MAin RESUlts}

In this section, we impose growth conditions on $f$ which allow us to apply Lemma 2.1 to establish some results of existence and multiplicity of positive solutions for BVP (1.1).

The following assumptions will be assumed throughout

$\left(H_{1}\right) f:[0, \infty) \rightarrow[0, \infty)$ is continuous, 
$\left(H_{2}\right) a(t)$ is nonnegative continuous in $(0, \mathrm{~T})$ and does not vanish identically on any subinterval, such that

$$
\int_{0}^{\frac{T}{2}} \int_{s}^{\frac{T}{2}} \varphi_{q}\left(\int_{0}^{r} \int_{R}^{\frac{T}{2}} a(x) \Delta x \Delta R\right) \Delta r \Delta s+\int_{\frac{T}{2}}^{T} \int_{\frac{T}{2}}^{s} \varphi_{q}\left(\int_{r}^{T} \int_{\frac{T}{2}}^{R} a(x) \Delta x \Delta R\right) \Delta r \Delta s<+\infty,
$$

here $\varphi_{q}(\omega)=|\omega|^{\frac{1}{p-1}} \operatorname{sgn}(\omega)$ is inverse function of $\varphi_{p}(s)$.

Then as $\delta \in(0, T / 2)$, we have $u(t) \geq \frac{\delta}{T}\|u\|$ by lemma 2.2. The function

$$
y(x):=\int_{\delta}^{x} \int_{s}^{x} \varphi_{q}\left(\int_{0}^{r} \int_{R}^{x} a(x) \Delta x \Delta R\right) \Delta r \Delta s+\int_{x}^{T-\delta} \int_{x}^{s} \varphi_{q}\left(\int_{r}^{T} \int_{x}^{R} a(x) \Delta x \Delta R\right) \Delta r \Delta s
$$

is positive and continuous in $[\delta, T-\delta]$.

Now we define an operator $\Phi: P \rightarrow P$

$W(t)=(\Phi u)(t):=$

$$
\left\{\begin{array}{l}
\int_{0}^{t} \int_{s}^{\sigma} \varphi_{q}\left(\lambda \int_{0}^{r} \int_{R}^{\sigma} a(x) f(u(x)) \Delta x \Delta R\right) \Delta r \Delta s, \quad t \in[0, \sigma], \\
\int_{t}^{T} \int_{\sigma}^{s} \varphi_{q}\left(\lambda \int_{r}^{T} \int_{\sigma}^{R} a(x) f(u(x)) \Delta x \Delta R\right) \Delta r \Delta s, \quad t \in[\sigma, T],
\end{array}\right.
$$

$\sigma$ is a solution of the following function

$$
\begin{aligned}
& \int_{0}^{x} \int_{s}^{x} \varphi_{q}\left(\lambda \int_{0}^{r} \int_{R}^{x} a(y) f(u(y)) \Delta y \Delta R\right) \Delta r \Delta s \\
= & \int_{x}^{T} \int_{x}^{s} \varphi_{q}\left(\lambda \int_{r}^{T} \int_{x}^{R} a(y) f(u(y)) \Delta y \Delta R\right) \Delta r \Delta s .
\end{aligned}
$$

With the monotonicity we known $\sigma \in(0, T)$ is existence and unique.

It is easy to check that $\Phi: P \rightarrow P$ is a completely continuous operator on $[0, \mathrm{~T}]$ by applying the Arzela-Ascoli theorem on time scales[3], and using Lebesgue's dominated convergence theorem on time scales[7].

$W(\sigma)$ is maximum of $W$ in $[0, T]$, because

$$
\begin{aligned}
& W^{\Delta}(\sigma)=0, \\
& W^{\Delta \Delta}(t)=\left\{\begin{array}{l}
-\varphi_{q}\left(\int_{0}^{t} \int_{R}^{\sigma} \lambda a(r) f(u(r)) \Delta r \Delta R\right) \leq 0, \quad t \in[0, \sigma], \\
-\varphi_{q}\left(\int_{t}^{T} \int_{\sigma}^{R} \lambda a(r) f(u(r)) \Delta r \Delta R\right) \leq 0, \quad t \in[\sigma, T] .
\end{array}\right.
\end{aligned}
$$

Now note $M=\min _{x \in[\delta, T-\delta]} y(x), N=\varphi_{q}\left(\int_{0}^{T} \int_{s}^{T} a(t) \Delta t \Delta s\right)$.

Theorem 3.1. Assume $\left(H_{1}\right)$ and $\left(H_{2}\right)$ are holding for $\lambda>0$, if there exists two different positive constants $h, k$ and $\delta \in\left(0, \frac{T}{2}\right)$ such that

$\left(A_{1}\right) f(u) \leq \frac{1}{\lambda}\left(\frac{h}{N}\right)^{p-1}, u \in[0, h]$,

$\left(A_{2}\right) f(u) \geq \frac{1}{\lambda}\left(\frac{2 k}{M}\right)^{p-1}, u \in[\delta k, k]$. 

$k$.

Then the BVP (1.1) has a positive solution $u$ such that $\|u\|$ is between $h$ and

Proof. Without loss of generality, we assume that $h<k$, consider the integral operator $\Phi$ defined as (3.1). We known the fixed point of $\Phi$ just is solution of (1.1). Now for complete the proof, divide it into two steps.

Step 1. Let $\Omega_{1}=\{u \in P:\|u\| \leq h\}$, for $u \in \partial \Omega_{1}$, by $\left(A_{1}\right)$

$$
\|\Phi u\|=\|W\|=W(\sigma) \leq \varphi_{q}\left(\int_{0}^{T} \int_{s}^{T} \lambda a(r) f(u(r)) \Delta r \Delta s\right) \leq h=\|u\|,
$$

so $\|\Phi u\| \leq\|u\|$ for $u \in \partial \Omega_{1}$.

Step 2. Let $\Omega_{2}=\{u \in P:\|u\| \leq k\}$, then for $u \in \partial \Omega_{2}$, there is $u(t) \in[\delta k, k]$, $t \in[\delta, T-\delta]$, therefore by condition $\left(A_{2}\right)$, if $\sigma \in(\delta, T-\delta)$, then

$$
\begin{aligned}
\|\Phi u\| & =\|W\|=W(\sigma) \\
\geq & \frac{1}{2}\left[\int_{0}^{\sigma} \int_{s}^{\sigma} \varphi_{q}\left(\lambda \int_{0}^{r} \int_{R}^{\sigma} a(x) f(u(x)) \Delta x \Delta R\right) \Delta r \Delta s\right. \\
& \left.+\int_{\sigma}^{T} \int_{\sigma}^{s} \varphi_{q}\left(\lambda \int_{r}^{T} \int_{\sigma}^{R} a(x) f(u(x)) \Delta x \Delta R\right) \Delta r \Delta s\right] \\
\geq & \frac{1}{2}\left[\int_{\delta}^{\sigma} \int_{s}^{\sigma} \varphi_{q}\left(\lambda \int_{\delta}^{r} \int_{R}^{\sigma} a(x) f(u(x)) \Delta x \Delta R\right) \Delta r \Delta s\right. \\
& \left.+\int_{\sigma}^{T-\sigma} \int_{\sigma}^{s} \varphi_{q}\left(\lambda \int_{r}^{T-\delta} \int_{\sigma}^{R} a(x) f(u(x)) \Delta x \Delta R\right) \Delta r \Delta s\right] \\
\geq & \frac{1}{2} \cdot \frac{2 k}{M} y(\sigma) \geq k=\|u\| .
\end{aligned}
$$

If $\sigma \geq T-\delta$, then

$$
\begin{aligned}
\|W\| & \geq \int_{0}^{T-\delta} \int_{s}^{T-\delta} \varphi_{q}\left(\lambda \int_{0}^{r} \int_{R}^{T-\delta} a(x) f(u(x)) \Delta x \Delta R\right) \Delta r \Delta s \\
& \geq \int_{\delta}^{T-\delta} \int_{s}^{T-\delta} \varphi_{q}\left(\lambda \int_{\delta}^{r} \int_{R}^{T-\delta} a(x) f(u(x)) \Delta x \Delta R\right) \Delta r \Delta s \\
& \geq 2 k>k=\|u\| .
\end{aligned}
$$

If $\sigma \leq \delta$, then

$$
\begin{aligned}
\|W\| & \geq \int_{\delta}^{T} \int_{\delta}^{s} \varphi_{q}\left(\lambda \int_{r}^{T} \int_{\delta}^{R} a(x) f(u(x)) \Delta x \Delta R\right) \Delta r \Delta s \\
& \geq \int_{\delta}^{T-\delta} \int_{\delta}^{s} \varphi_{q}\left(\lambda \int_{r}^{T} \int_{\delta}^{R} a(x) f(u(x)) \Delta x \Delta R\right) \Delta r \Delta s \\
& \geq \frac{2 k}{M} y(\sigma)>2 k>k=\|u\| .
\end{aligned}
$$

So we conclude that $\|\Phi u\| \geq\|u\|$ for $u \in \partial \Omega_{2}$. Then according to the Lemma 2.1 , this complete the proof.

Corollary 3.2. If $\left(H_{1}\right)$ and $\left(H_{2}\right)$ hold, and $f_{\infty}=f_{0}=+\infty$, then there exist $\lambda^{*}>0$ such that for every $\lambda \in\left(0, \lambda^{*}\right), B V P(1.1)$ have two positive solutions. 
Proof. Let $\lambda^{*}=\sup _{h^{*}>0}\left[1 / B\left(h^{*}\right)\right]$, where $B\left(h^{*}\right):=\left[h^{*} / N\right]^{1-p} \cdot \sup _{u \in\left[0, h^{*}\right]} f(u)$ for $\lambda \in$ $\left(0, \lambda^{*}\right)$, there is $\bar{h}>0$, such that $\lambda \leq[1 / B(\bar{h})]$. Furthermore,

$$
f(u) \leq \frac{1}{\lambda}\left(\frac{\bar{h}}{N}\right)^{p-1}, u \in[0, \bar{h}] .
$$

This satisfies assumption $\left(A_{1}\right)$ of Theorem 3.1.

On the other hand, because $f_{\infty}=f_{0}=+\infty$, there exists $k_{1}, k_{2}>0, k_{1}>\bar{h}>k_{2}$ such that

$$
\begin{aligned}
& \frac{f(u)}{u^{p-1}} \geq \frac{1}{\lambda}\left(\frac{2}{M}\right)^{p-1}, u \in\left[\delta k_{1}, k_{1}\right] \subseteq\left[\delta k_{1},+\infty\right), \\
& \frac{f(u)}{u^{p-1}} \geq \frac{1}{\lambda}\left(\frac{2}{M}\right)^{p-1}, u \in\left[\delta k_{2}, k_{2}\right] \subseteq\left(0, k_{2}\right] .
\end{aligned}
$$

Then $k_{1}, k_{2}$ satisfy the assumption $\left(A_{1}\right)$ of Theorem 3.1 , respectively. This complete the proof.

Corollary 3.3. If $\left(H_{1}\right)$ and $\left(H_{2}\right)$ hold, and $f_{\infty}=f_{0}=0$, then there exist $\lambda^{\prime}>0$ such that for every $\lambda \in\left(\lambda^{\prime},+\infty\right), B V P$ (1.1) have two positive solutions.

Proof. Let $\lambda^{\prime}=\inf _{k^{\prime}>0}\left[\left(k^{\prime}\right)^{(p-1)} / A\left(k^{\prime}\right)\right]$, where $A\left(k^{\prime}\right):=(2 / M)^{1-p} \cdot \inf _{u \in\left[\delta k^{\prime}, k^{\prime}\right]} f(u)$, because $\lambda>k^{\prime}$, so there exists $\bar{k}$ such that $\lambda>\left[\bar{k}^{p-1} / A(\bar{k})\right]$. So

$$
f(u)>\frac{1}{\lambda}\left(\frac{2 \bar{k}}{M}\right)^{p-1}, u \in[\delta \bar{k}, \bar{k}] .
$$

This satisfies the assumption $\left(A_{2}\right)$ of Theorem 3.1.

On the other hand, by $f_{\infty}=f_{0}=0$, combined the continuity of $f$ we known there exist two constants $h_{1}, h_{2}>0, h_{1}<\bar{k}<h_{2}$ such that

$$
\begin{aligned}
& \frac{f(u)}{h_{1}^{p-1}} \leq \frac{1}{\lambda} N^{1-p}, u \in\left[0, h_{1}\right], \\
& \frac{f(u)}{h_{2}^{p-1}} \leq \frac{1}{\lambda} N^{1-p}, u \in\left[0, h_{2}\right] .
\end{aligned}
$$

(We are able to do it because if let $f^{*}(u)=\max _{t \in[0, u]} f(t)$, then $f_{\infty}=0 \Leftrightarrow f_{\infty}^{*}=$ $0)$. Then $h_{1}, h_{2}$ satisfy the assumption $\left(A_{2}\right)$ of Theorem 3.1, this complete the proof.

It is easy to acquire the following conclusion according the proves of corollary (3.1) and (3.2).

Corollary 3.4. Assume $\left(H_{1}\right)$ and $\left(H_{2}\right)$ hold, if $f_{\infty}=\infty, f_{0}=0$ or $f_{\infty}=0$, $f_{0}=\infty$, then for every $\lambda \in(0,+\infty)$, BVP (1.1) has a positive solution. 


\section{REFERENCES}

1. R.I. Avery, D.R. Anderson, Existence of three positive solutions to a second-order boundary value problem on a measure chain, J. Comput. Appl. Math. 141 (2002), 65-73.

2. R.P. Agarwal, M. Bohner, D. ORegan, A. Peterson, Dynamic equations on time scales: A survey, J. Comput. Appl. Math. 141 (2002), 1-26.

3. R.P. Agarwal, M. Bohner and P. Rehak, Half-linear dynamic equations, Nonlinear Analysis and Applications: To V. Lakshmikantham on his 80th Birthday vol. 1, Kluwer Academic Publishers, Dordrecht, 2003, pp.1-57.

4. D.R. Anderson, J.M. Davis, Multiple solutions and eigenvalues for third-order right focal boundary value problem, J. Math. Anal. Appl. 267 (2002), 135-157.

5. G.A. Afrouzi, M.K. Moghaddam, J. Mohammadpour and M. Zameni, On the positive and negetive solutions of Laplacian BVP with Neumann boundary conditions, J. Nonlinear Sci. Appl. 2 (2009), 38-45.

6. R.P. Agarwal, H. L and D. ORegan, Existence theorem for the one-dimensional singular p-Laplacian equation with sign changing nonlinearities, Appl. Math. Comput. 143 (2003), $15-38$.

7. B. Aulbach, L. Neidhart, Integration on measure chains, in: Proc. of the Sixth Int. Conf. on Difference Equations, CRC, Boca Raton, FL, 2004, pp.239-252.

8. R.P. Agarwal, D. ORegan, Nonlinear boundary value problems on time scales, Nonlinear Anal. 44 (2001), 527-535.

9. Z.B. Bai, The upper and lower solution method for some fourth-order boundary value problems, Nonlinear Anal. 67 (2007), 1704-1709.

10. Z.B. Bai, Z. Du, Positive solutions for some second-order four-point boundary value problems, J. Math. Anal. Appl. 330 (2007), 34-50.

11. Z.B. Bai, X.Q. Liang, Z.J. Du, Triple positive solutions for some second-order boundary value problem on a measure chain, Comput. Math. Appl. 53 (2007), 1832-1839.

12. X. Dong, Z.B. Bai, Positive solutions of fourth-order boundary value problem with variable parameters, J. Nonlinear Sci. Appl. 1 (2008), 21-30.

13. M. Bohner, A. Peterson, An Introduction with Applications, Dynamic Equations on Time Scales, Birkhäuser, Boston, 2001.

14. S.Hilger, Analysis on measure chains-a unified approach to continuous and discrete calculus, Results Math. 4 (1990), 18-56.

15. M.A. Krasnosel'skii, Positive Solutions of Operator Equations, Noordhoff, Gronignen, 1964.

16. R.Y. Ma, H.Y. Wang, On the existence of positive solutions of fourth-order ordinary differential equations, Applicable Analysis. 59 (1995), 225-231.

17. H.R. Sun, W.T. Li, Multiple positive solutions for p-Laplacian m-point boundary value problems on time scales, Appl. Math. Comput. 182 (2006), 478-491.

18. H.R. Sun, W.T. Li, Existence theory for positive solutions to one-dimensional p-Laplacian boundary value problems on time scales, J. Differential Equations. 240 (2007), 217-248.

19. J.Y. Wang, The existence of positive solutions for the one-dimensional $p$-Laplacian, Proc. Amer. Math. Soc. 125 (1997), 2275-2283.

20. Y.Z. Zhu, J. Zhu, Several existence theorems of nonlinear m-point boundary value problem for p-Laplacian dynamic equations on time scales, J. Math. Anal. Appl. 344 (2008), 616626 .

1 College of Information Science and Engineering, Shandong University of Science and Technology, Qing Dao, 266510, P. R. China.

E-mail address: pyy851@hotmail.com

2 College of Information Science and Engineering, Shandong University of Science and Technology, Qing Dao, 266510, P. R. China.

E-mail address: zhanbingbai@163.com 